\title{
Critical Evaluation of Current Concepts and Moving to New Horizons in the Management of IBD
}

Relevant progress has been made in unraveling the etiopathogenesis of chronic inflammatory bowel diseases (IBDs) in the past years, and the diagnostic and therapeutic approaches to IBD patients have been changed recently or will be changing significantly in the near future. The relevance of well-established IBD therapies has recently been critically re-assessed using principles of evidencebased medicine, and highly sophisticated evidence-based consensus guidelines have been developed in order to standardize and improve our current diagnostic and therapeutic approaches. On the other hand, various new therapeutic options have emerged and are integrated into our treatment algorithms. The clinical relevance of these new modalities is sometimes difficult to assess for physicians who do not frequently treat the full spectrum of IBD patients.

These proceedings aim to critically discuss established and emerging new concepts in the pathogenesis, diagnosis and treatment of IBD reflecting the recent advances in the etiopathogenesis of IBD. The content of these proceedings has been presented and intensely discussed in an international symposium in Frankfurt from March 6-7, 2015, entitled 'Critical Evaluation of Current Concepts and Moving to New Horizons in the Management of
IBD'. Controversial problems, such as the optimal diagnostic and therapeutic strategy, optimal control and selection of established therapies as well as the integration of novel IBD therapies into our current treatment algorithms were addressed and critically discussed. Special emphasis has been directed at the multidisciplinary management of complex IBD patients. Difficult therapeutic situations were analyzed and discussed by international experts. In addition, relevant aspects of the current 'omics' era of research for IBD clinicians were highlighted and their relevance in the future management of IBD patients were discussed.

The editor likes to thank all contributors for their timely manuscripts, which made rapid publication of the proceedings possible. These contributions certainly reflect the current state of the art in the etiopathogenesis, diagnostic approach and treatment of patients with IBD. The editor is indebted to the Falk Foundation, Freiburg, for their generous support and the exceptional organization of the meeting. Furthermore, I wish to thank Brigitte Thierstein and her colleagues from S. Karger, Switzerland, for their help and cooperation in preparing this issue.

Axel Dignass, Frankfurt 